\title{
Original Research \\ Communication Strategies and Timeliness of Response to Life Critical Telemetry Alarms
}

\author{
Kimberly A. Bonzheim, M.S.A., Rani I. Gebara, M.S., ${ }^{2}$ \\ Bridget M. O'Hare, M.S., ${ }^{2}$ R. Darin Ellis, Ph.D., ${ }^{3}$ \\ Monique A. Brand, B.A., ${ }^{1}$ Salil D. Balar, M.S. ${ }^{2}$ \\ Rita Stockman, M.S.N., ${ }^{1}$ Annette M. Sciberras, B.S.N., ${ }^{1}$ \\ and David E. Haines, M.D. ${ }^{1}$ \\ ${ }^{1}$ Department of Cardiovascular Medicine, Beaumont Hospital, \\ OUWB School of Medicine, Royal Oak, Michigan. \\ ${ }^{2}$ Department of Biomedical Engineering, Beaumont Hospital, \\ Royal Oak, Michigan. \\ ${ }^{3}$ Industrial \& Systems Engineering Department, Wayne State \\ University, Detroit, Michigan.
}

\begin{abstract}
Background: A centralized electrocardiogram telemetry monitoring system (TMS) facilitates early identification of critical arrhythmias and acute medical decompensation. Timely intervention can only be performed if abnormalities are communicated rapidly to the direct caregiver. The study objectives were to measure effectiveness of bidirectional voice communication badges versus one-way alphanumeric pagers for telemetry alarm response and communication loop closure. Methods: A sequential observational pilot study of nursing response to TMS alarms compared communication technologies on four nursing units in a 1,061 bed tertiary care hospital with 264 TMS channels of telemetry over a 2-year period. Subsequently, the communication technologies were compared in a randomized fashion on a 68-bed progressive cardiac care unit. Caregivers were blinded to the protocol. All alarm responses were recorded during two periods using either pagers or voice communication devices. Alarm response time and closure of the communication loop were analyzed in a blinded fashion. Results: The direct communication functionality of the badge significantly shortened the time to first contact, time to completion, and rate of closure of the communication loop in both the pilot and study phases. Median time to first contact with the communication badge was $0.5 \mathrm{~min}$, compared to $1.6 \mathrm{~min}$ with pager communication ( $p<0.0003)$. Communication loop closure was achieved in 100\% of clinical alarms using the badge versus 19\% with the pager ( $1<0.0001)$. Conclusions: Communication badge technology reduced alarm time to first contact and completion as well as facilitated communication loop closures. Immediate two-way communication significantly impacted practice, alarm management, and resulted in faster bedside care.
\end{abstract}

Key words: cardiology/cardiovascular disease, information management, telecommunications

\section{Introduction}

S

uccessful resuscitation from tachyarrhythmic cardiac arrest increases with reduction of time to defibrillation. ${ }^{1-5}$ In a hospital setting, a centralized electrocardiogram (ECG) telemetry monitoring system (TMS) may be employed to facilitate early identification of critical arrhythmias and acute medical decompensation to prevent an emergent episode. ${ }^{5-9}$ Over the last 40 years, the intelligence of the monitoring equipment has significantly improved to include arrhythmia identification algorithms, monitoring for myocardial ischemia, assessment of abnormal repolarization (QT interval), artifact reduction, and multilead monitoring capabilities. ${ }^{10}$ Therefore, the role of specially trained central telemetry technicians has expanded beyond observing heart rates and basic rhythms to interpreting complex arrhythmias, identifying ischemic ECG changes, and detecting prolonged QT intervals. Even with these technological advances, the need for human validation and interpretation of telemetry alarms is as important today as it was 40 years ago. ${ }^{10,11}$ In a centralized TMS model, the expertise and focus of the monitoring technician is employed to assist nurses who may not have the experience/skills required for advanced heart rhythm interpretation, ${ }^{10}$ nor the time or inclination to carefully observe the monitor. A centralized TMS system frees the nurse to attend to the needs of the patient rather than watch the monitor continuously at the patient's bedside. In addition, alarm fatigue and desensitization plague clinical environments and negatively affect patient outcomes, but a centralized TMS system precludes the need for monitor alarms on the patient unit. Therefore, a central TMS with a reliable immediate communication process can be an effective model for ECG alarm management.

After alarm detection, appropriate interventions to protect patients can only be effected if vital information is communicated rapidly and efficiently from the central monitoring station to the direct caregiver in the patient care setting. Despite the critical nature of this communication, no scientific data exist that assess differing communication modes and their relative effectiveness. Modalities of critical telemetry alarm communication between caregivers include one-way communication devices such as alphanumeric pagers (text messaging), immobile dedicated land line phones, cell phones, ${ }^{12}$ and new bi-directional voice communication technologies. ${ }^{13}$ In the present study, the value of intensive nurse training for alarm response was tested over time, and competing communication technologies were compared. It was hypothesized that replacement of one-way communication devices (alphanumeric pagers) with bi-directional voice communication devices would facilitate the information hand-off between caregivers in a centralized TMS. It 


\section{BONZHEIM ET AL.}

should also improve the alarm response time to the patient due to an increased likelihood of completing the communication loop, ultimately enhancing alarm management.

\section{Methods}

\section{SETTING AND SUBJECTS}

The study was conducted at a 1,061-bed level 1 trauma center teaching facility with patients with telemetry spread throughout 2 million square feet of the hospital on 14 different nursing units. Specially trained technicians are housed in a central telemetry station that includes five monitoring stations of 48-72 patients per technician station for a total of 264 channels (Patient Net and ApexPro; GE Healthcare, Milwaukee, WI). The technician's role is to validate alarms as they occur and communicate true alarms per protocol to the appropriate nurse taking care of the patient on the floor. The nurses have ECG rhythm viewing stations available at their respective nursing stations to view a patient's rhythm if necessary, and the medical/surgical units have opted to turn off the audible alarms on their nursing units. The availability of bedside monitors on the noncritical care wards is limited. This centralized TMS model eliminates the need to staff a monitor watcher at each nursing station and allows nurses to leave the patients' room and their bedside monitors to attend to other duties. It also eliminates the scenario that requires an untrained unit secretary to listen for monitor alarms when the nurses are busy providing bedside care.

\section{COMMUNICATION TECHNOLOGIES AND PROCESSES}

The legacy technology employed one-way alphanumeric pagers. The technician responsible for verifying each alarm initiated the process of selectively paging the group of nurses assigned to the pager according to predetermined policy criteria. Alarms were categorized by the technician as level 1, 2 (clinical alarms), or 3 (technical alarms) according to the criteria shown in Table 1. All levels of alarms generated a page to the caregiver, and critical level 1 alarms also triggered a phone call to the unit secretary to announce the alarm overhead. The communication loop was closed when the nurse returned a confirmatory call to the TMS technician. If the nurse did not respond within $3 \mathrm{~min}$, the technician was required to re-page the alarm every $3 \mathrm{~min}$, elevating it to include nursing management, until the communication loop was closed and the information hand-off was complete.

The comparison communication technology introduced was a voice-activated communication badge (Vocera Communications, Inc., San Jose, CA) that the caregiver would wear clipped to his/her clothing or on a lanyard around the neck. The two-way voice communication badge was initially selected due to its unique characteristics including hands-free operation, voice activation, two-way closed loop communication, human-to-human information handoffs, immediate ability to locate the patient's caregiver, and automated escalation pathways to other caregivers if necessary. After alarm recognition, the communication badge was voice-activated by the technician who called out the patient's room number. The system automatically routed the call to the badge of the patient's caregiver

\begin{tabular}{|c|c|c|}
\hline PRIORITY & $\begin{array}{l}\text { ALARM ON } \\
\text { THE PAGER }\end{array}$ & ALARM DEFINITION \\
\hline \multirow[t]{4}{*}{1} & Asystole & Asystole $>3 \mathrm{~s}$ \\
\hline & $V$-fib & V-fib \\
\hline & V-tach & $V$-tach $\geq 10$ beats or $150 \mathrm{bpm}$ \\
\hline & Low HR & Low heart rate $<40 \mathrm{bpm}$ \\
\hline \multirow[t]{9}{*}{2} & V-tach & $V$-tach $<10$ beats and heart rate $<150$ \\
\hline & V-rhythm & $\begin{array}{l}\text { V-rhythm } \geq 8 \text { beats and heart rate } \\
<120 \text { bpm }\end{array}$ \\
\hline & V-run & V-run 4-8 beats regardless of heart rate \\
\hline & \multirow[t]{3}{*}{ High HR } & High heart rate $>130$ bpm \\
\hline & & New onset Afib \\
\hline & & SVT lasting $>30 \mathrm{~s}$ \\
\hline & \multirow[t]{3}{*}{ Low HR } & Low heart rate $<50$ bpm \\
\hline & & New onset heart block \\
\hline & & New onset pacemaker malfunction \\
\hline \multirow[t]{8}{*}{3} & \multirow[t]{2}{*}{$L D$ off (RA, LA, RL, LL) } & Patient not monitored \\
\hline & & One or more leads off \\
\hline & \multirow[t]{2}{*}{ No Signal } & Patient not monitored \\
\hline & & ECG rhythms cannot be seen \\
\hline & \multirow[t]{2}{*}{ Muscle } & Patient not monitored \\
\hline & & Muscle artifact is present \\
\hline & \multirow[t]{2}{*}{ No arrhythmia } & Patient not monitored \\
\hline & & $\begin{array}{l}\text { ECG signal is uninterruptible to com- } \\
\text { puter's arrhythmia software }\end{array}$ \\
\hline
\end{tabular}

$E C G$, electrocardiogram; bpm, beats per minute; $L A$, left arm; LD, lead; LL, left leg; $R A$, right arm; RL, right leg; SVT, supraventricular tachycardia.

who had logged into the system at the beginning of the shift. The caregiver could verbally accept the call, at which time the technician could relay the alarm information directly to the caregiver and the caregiver could query the technician regarding current alarm status and rhythm histories for that patient. With this verbal interchange, the communication hand-off was completed and confirmed. If the call was unanswered by the primary nurse, it was automatically transferred within seconds to another nurse in the Nurse's Unit group. If necessary, the communication badge system would continue to escalate the call to the Unit Charge Nurse, the Nurse Manager, and finally the Unit Secretary's desk phone.

\section{PILOT PROTOCOL}

Before the study, a pilot implementation to replace the alphanumeric pagers with the new communication badge technology was 


\section{VOICE COMMUNICATION AND ECG TELEMETRY}

initiated on a 45-bed Oncology Unit that has a low volume of TMS usage. Subsequently, the communications technology was introduced on three high volume TMS units. The nurses on these units comprised the observation group and were compared in a non randomized fashion to nurses on units using the legacy pager technology. In the initial pilot phases, observers were trained to identify the exact time of an alarm, the time of caregiver notification(s), and the time of caregiver communication to the technician or resolution of leads off or other technical issues. The observers prospectively recorded these data at the TMS stations during predefined observation sessions of $2 \mathrm{~h}$ a day, and data were compared between caregivers using alphanumeric pagers versus those with the voice communication badges. To test the value of training and documentation of competency toward a newly revised standardized Telemetry/Nursing TMS policy, a training blitz was performed for all nursing and telemetry technician staff. Performance measures were tested with a written test before the pilot study and repeated quarterly throughout the pilot period. Comparisons of alarm response times and communication loop closures were made before and after the training blitz and between communication technologies.

\section{STUDY PROTOCOL}

Alarm response time data were prospectively collected. The study subjects were the 18-20 nurses who staff a 68-bed progressive care cardiology unit with a ratio of 3 to 4 patients per staff member. The voice communication technology was sequentially introduced to three nursing stations on this unit at the initiation of the protocol (one station with communication badges and two stations with pagers), after 2 weeks (two stations with communication badges and one station with pagers), and after study conclusion. Thus, during the 4-week study period, 6 station-weeks employed communication badges and 6 station-weeks employed pagers. At the beginning of each shift, the caregivers (nurses and nurse assistants) were randomly assigned either an alpha numeric pager or a communication badge for alarm notification for that day by assigning them to a station with or without the new technology. Randomization was achieved by the nursing director drawing assignment numbers from a hat and then passing these assignments on to the charge nurses. Nurses were not informed of the randomized nature of their daily coverage assignments. Specially trained observers collected data Monday-Friday for $7.5 \mathrm{~h}$ per day for 4 consecutive weeks. One observer was stationed at the monitor station in the TMS department, and a second observer was stationed on the nursing unit to discreetly document the time the notified caregiver crossed the threshold of the patient's room for the clinical alarms.

The response times for all alarm levels for either alphanumeric paging or two-way voice communication during the data collection times were documented. Time to first contact was defined as the time measured from initial activation of the communication attempt by the TMS technician to the successful hand-off of clinical information to the caregiver on the floor with confirmation of receipt of the information, thus closing the communication loop. Time to alarm completion for clinical alarms (arrhythmias) was defined as the time measured from initial activation of the communication attempt to the time that the caregiver crossed the threshold into the patient's room. Given the low frequency of these alarms, simultaneous alarms with two or more patients did not occur during the study period. Time to alarm completion for technical alarms (leads off) was defined as the time measured from initial activation of the communication attempt to the time that the technical problem was rectified (e.g., leads reattached). The observers synchronized their digital watches to the clock in the telemetry system to standardize the time records. Alarm response times reflect all aspects of the process, including time spent sending multiple pages, contacting nurse managers, and waiting for return phone calls from the nurse to the TMS technician. If there was no verbal response to the alarm by the caregiver but the alarm condition was addressed or corrected, the time to first contact was defined as the same time as the time to completion. Alarms with incomplete data at the end of the daily data collection were censored from the time analysis. The number of attempts that the telemetry technician needed to contact the nurse includes the number of pages, phone calls, and communication badge calls necessary to complete the alarm.

\section{DATA ANALYSIS}

The data collection was obtained by a small specially trained group of observers, and the caregivers were not informed about the collection of alarm response data until completion of the study so that response bias would be minimized. All data were prospectively collected, de-identified as to the name of the patient and caregiver, and stored in a computerized file. Data are expressed as means \pm standard deviations for normally distributed data and medians and ranges for non-normal data. In the pilot phase, continuous data were compared using unpaired two-tailed $t$-tests. Categorical data were compared with Fishers exact test. In the study protocol, the measurements were analyzed using Wilcoxon rank tests comparing pager to communication badge. The type of alarm and number completed were examined using Pearson's chi-square tests. An alpha level of $<0.05$ was considered significant.

\section{Results}

\section{RESPONSE TIMES TO CLINICAL ALARMS}

During the communication badge pilot protocol, a total of 482 clinical alarms were recorded during the direct observation periods, 258 with alphanumeric pagers and 229 with the communication badge. Using alphanumeric pagers, communication loops were closed with verbal confirmation in only $26 \%$ of events. In 209 cases, where the communication loop closure could be confirmed (either verbal confirmation or evidence of correction of technical problem such as lead disconnection without verbal confirmation), the median alarm hand-off/response times using the alphanumeric pager for communication was $6.5 \mathrm{~min}$ (interquartile range [IQR] 2.0-14.0 min). In comparison, the 229 alarms recorded using two-way voice communication had a successful closure of the communication loop in $100 \%$ of events ( $p<0.0001$, Fig. 1). The median alarm response time to successfully hand-off information was $0.6 \mathrm{~min}$ (IQR 0.5-0.8 $\mathrm{min}$, $p<0.0001)$. 


\section{BONZHEIM ET AL.}

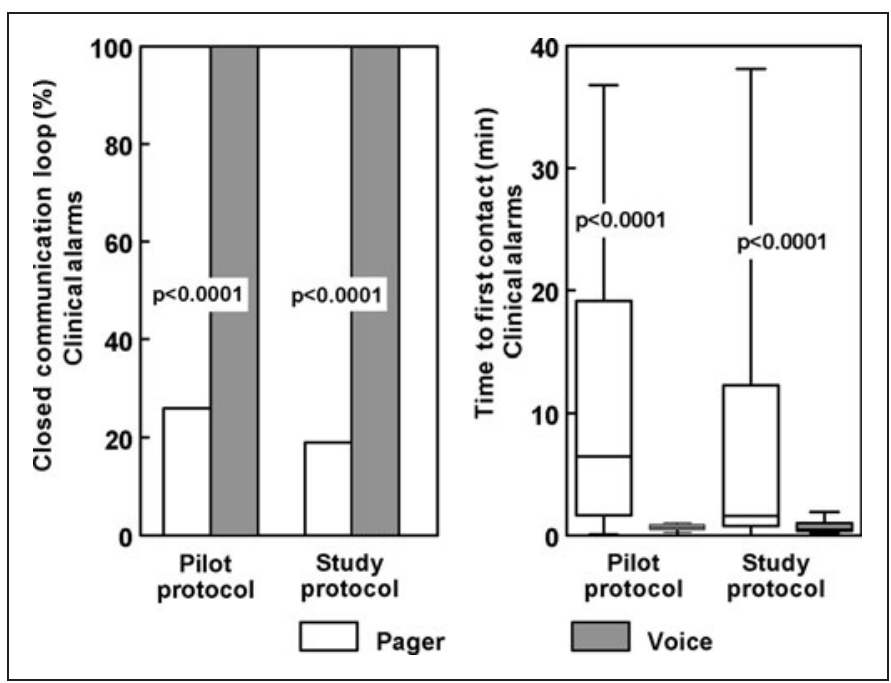

Fig. 1. The prevalence of successful closure of the communication loop for one-way alphanumeric pager communication (open bar) versus two-way voice communication (gray bar) is shown in the left graph. The right graph shows a box and whisker plot of the time to first contact in minutes for all level 1 and 2 clinical alarms in the pilot and study protocols. The boxes represent the $25^{\text {th }}$ to $75^{\text {th }}$ percentile with the median value shown with a horizontal line. The whiskers represent the data range.

During the communication badge randomized protocol, the nurse to patient ratio on the study unit averaged 1:3.5, with an average daily unit occupancy of 91\% (62 patients/68 beds). A total of 811 alarms were evaluated ( $n=499$ pager; $n=312$ badge), of which 55 were level 1 or 2 clinical alarms ( $n=32$ pager; $n=23$ badge). The median time to first contact for level 1 or 2 alarms with the communication badge was $0.5 \mathrm{~min}$ (IQR $0.4-0.7 \mathrm{~min}$ ), compared with $1.6 \mathrm{~min}$ (IQR $1.0-3.7 \mathrm{~min}$ ) with pager communication $(p<0.0003$ ). The number of attempts needed to complete the alarm was significantly lower in the badge group (1.3 \pm 0.7 attempts/alarm) compared with the pager process $(2.1 \pm 1.6$ attempts/alarm, $p<0.0001)$. Communication loop closure was achieved in only $19 \%$ of clinical alarms with pager communication compared with 100\% of alarms using the communication badge $(p<0.0001)$. Despite more rapid, complete, and informative communication with the communication badge, the median time to level 1 or 2 alarm completion was similar between groups (1.6 min; IQR 1.1-3.8 for pagers vs. $1.9 \mathrm{~min}$; IQR 0.95.5 for communication badges, $p=0.77$ ). Paradoxically, task completion defined by the nurse crossing the threshold to the patient's room was achieved in 97\% of those using pagers but only $61 \%$ of those with communication badges ( $p=0.001$ ). Thus, in 39\% of level 1 or 2 alarms where the full context and content of the alarm information could be discussed between the technician and caregiver, there was no visit to the patient's room.

\section{RESPONSE TIMES TO TECHNICAL ALARMS}

Throughout the study period, alarm response time for technical alarms (determined complete when the leads were reattached) were recorded. Technical alarms account for the majority of alarms (93.6\%) and are a major cause of alarm desensitization. A total of 756 technical alarms were evaluated, and it was observed that the nurses responded to leads off alarms faster when the telemetry technician used the communication badge to communicate. The median time to alarm completion with reattachment of the leads was $8.7 \mathrm{~min}$ (IQR 3.5-18.3) with the pager compared with $3.4 \mathrm{~min}$ (IQR 1.1-10.2) with the communication badge ( $p<0.0001)$.

\section{RESULTS OF STAFF TRAINING}

The pager alarm response times on all units from January 2005 to the end of 2006 that bridged the periods of staff retraining are summarized in Figure 2. There was no significant change in pager response time regardless of the pager process standardization efforts and the mandatory education competency blitzes. Similarly, the communication badge response times did not show a significant change throughout the observation quarters. When all communication badge alarm levels were analyzed in 2006, including addressing the level 3 technical alarms, the improvement in alarm response time among study quarters in 2006 using the communication badge technology was sustained. Since 2006, there have been no sentinel events or known near misses related to alarm communication using the communication badge, compared with a previous period of similar duration where two near misses and one sentinel event occurred.

\section{Discussion}

In the present study, the alarm communication process using the one-way communication of an alpha-pager was compared with a new bi-directional communication badge. Alarm response time was employed as a measure of the clinical information hand-off between a telemetry technician and a nurse. The time to first contact and

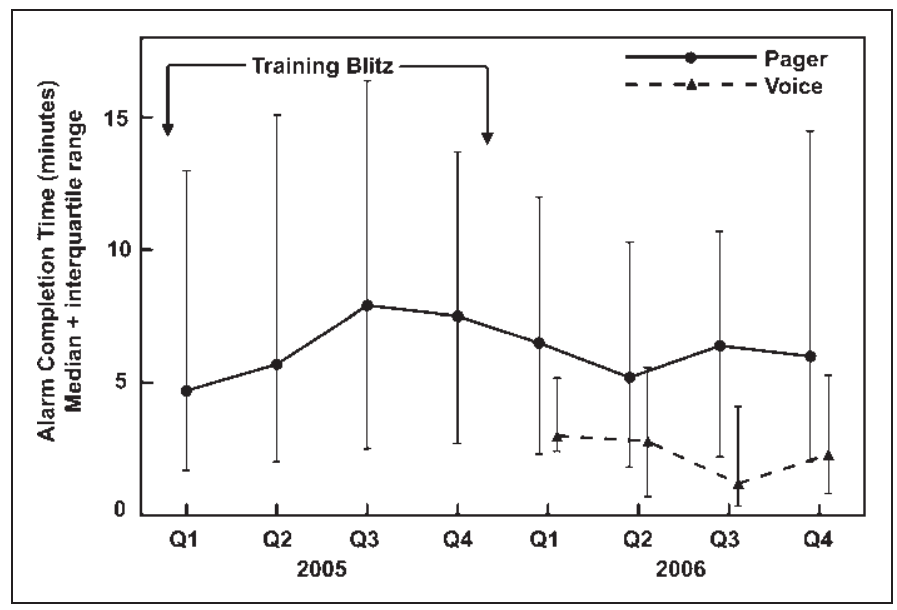

Fig. 2. Alarm completion times from the Pilot for all alarms in the alphanumeric pager test group (solid line) and the two-way voice communication group (broken line) versus calendar quarter. Vertical lines represent the $25^{\text {th }}$ to $75^{\text {th }}$ percentile values. Training blitzes immediately preceded the first quarter of 2005 and were repeated again before the first quarter of 2006. 


\section{VOICE COMMUNICATION AND ECG TELEMETRY}

minutes to alarm completion were measured. Significant improvement in all metrics except time to alarm completion was seen when the communication badge was incorporated into the process. Response times to both clinical and technical alarms were significantly reduced. Due to the design of the communication badge, all communications with this technology resulted in communication loop closure. Message delivery with confirmation of message receipt was a forced function and guaranteed improved communication. In contrast, the failure to reliably close communication loops in the pager process reflected the extra steps necessary for the nurse to call the telemetry technician to confirm receipt of the alarm page. One-way communication with pagers does not provide the necessary force function for this critical communication process to work. The observed improvements in communication were a result of the new communication technology and not due to a renewed emphasis on the communication process, as evidenced by the lack of improvement of our metrics after repeated training blitzes.

The bi-directional voice communication device performed in our process as anticipated. Closure of the communication loop was a forced function, resulting in 100\% completion of this important metric. The steps required to send or receive alarm notification were reduced with the communication badge compared with the pager. Fewer steps in the process increased the likelihood of task completion by minimizing opportunities for interruptions or distractions that often result in failure to complete the initial task. This resulted in a significant reduction in the median time to alarm hand off. The real-time verbal hand-off helped prevent miscommunications and misunderstandings that might otherwise occur, as an opportunity to ask and respond to questions was available when the communication badge was used. In the study, the communication badge minimized the number of repeat alarm calls sent, which would be anticipated to lessen or prevent alarm desensitization. It is likely that nurses had increased confidence that an alarm communicated by a human voice with its intonations was true and valid.

The more accurate and timely information hand-off with the communication badge did not result in a reduction of the median time to level 1 and 2 alarm completion (the primary endpoint), as measured by the time from alarm notification to the caregiver crossing the threshold of the patient's room, but it did result in a significantly lower prevalence of room entry by the nurse compared with the alphanumeric pager. A possible explanation for this outcome is that the direct voice communication resulted in more complete information transfer to the caregiver. This would mitigate the requirement for the caregiver to directly assess the patient and delay entry into the patient's room if the TMS observations were assessed as nonthreatening to the patient or were anticipated based on earlier observations. The information discussed verbally using the communication badge helps the nurse to better prioritize her time and the multitasking that encompasses her day. In contrast, non-verbal communication of an alarm occurrence likely triggered a direct patient evaluation promptly in most cases to rule out potentially dangerous arrhythmias or a serious clinical event.

\section{UTILITY OF IMPROVED TELEMETRY ALARM COMMUNICATION}

Almost no scientific data examining the process of telemetric monitoring, its cost, or its value exists despite its ubiquitous application in the inpatient setting. Chan et al. demonstrated that inpatients on telemetry had a 53\% risk reduction for delayed defibrillation compared with those who were unmonitored and that both survival and neurological outcomes were directly related to time of defibrillation. ${ }^{4}$ The communication process employed in the present trial is compliant with the Joint Commission's National Patient Safety Goal (Requirement \#2E), first published in 2006, that standardizes the hand-off of communications. ${ }^{14}$ In addition, the communication badge emulates the established nursing behavior of patient information hand-off between caregivers. The ability to be immediately aware of a change in a patient's clinical condition allowed the nurse to better prioritize patient care efforts. Supporting a timely closure of the alarm allowed both the TMS technicians and the nurses to focus on the priorities at hand rather than having incomplete alarm notifications waiting for a confirmation call. The time to medical intervention and/ or defibrillation in a critically ill patient is dependent on multiple factors, only one of which is the time from event onset to notification of the caregiver. Despite the fact that the times to first contact and alarm completion are surrogate endpoints for actual medical intervention, it follows that faster notification of the caregiver should lead to faster delivery of critical care. Therefore, use of the communication badge could positively influence patient outcomes.

Best practices confirmed the trends identified in the data set. Alarm fatigue and desensitization are common problems across the country. ${ }^{15,16}$ In relevant studies, clinicians were found to react by ignoring pagers and messages or waiting until they were paged twice before responding, to separate trivial from the truly urgent. ${ }^{17}$ Moreover, verbal communication is a more efficient means of rapidly conveying information, requiring less reliance on cognitive resources and attention. ${ }^{18}$ False positive nuisance alarms are major contributors to alarm fatigue and necessitate better alarm management. Some patients generate frequent false positive alarms. ${ }^{19} \mathrm{~Pa}-$ tients who in the past were monitored while lying still in bed are now more mobile. When nurses respond to false alarms, it diverts their attention and time from their other critical duties and reinforces alarm desensitization and frustration. An alarm management process can only work in a system where the number of false positive alarms has been decreased to maintain the validity and value of the alarm notification. Human oversight and decision support provided by highly trained telemetry technicians and improved equipment alarm intelligence will further enhance the true positive rates. Alarms should be truly actionable to improve patient safety. ${ }^{18}$

\section{Limitations}

The manual process required to collect alarm response time data was subject to human error. However, any error would be expected to be equally applicable to both groups. Data collection was limited to daytime during the week. It is possible that observations during nights and weekends might have yielded different results. Since 


\section{BONZHEIM ET AL.}

monitoring alarm response was a labor-intensive process, the number of alarms evaluated was limited. The limited number of level 1 and 2 alarms in this study did not provide adequate power to assess the effect of the voice communication badge on hard endpoints such as survival after in-hospital cardiac arrest. In addition, technical alarms were the only alarm types that provided a measurable endpoint of positive action toward patient care (i.e., lead re-attachment time). The presence of observers on the floor could have biased the activities of the nurses being observed, although it is anticipated that that effect would have equal weight between the two groups. The time to alarm completion is a surrogate endpoint and does not actually represent the time to care delivery.

\section{Conclusion}

The use of the communication badge technology markedly reduced alarm hand-off/response time and facilitated communication loop closures. The inherent features of the two-way communication badge including voice-activation, hands-free operation, and an automated escalation pathway had a significant impact on alarm management for the nursing areas. The bi-directional alarm handoffs gave the nurses an opportunity to quickly ask questions and confirm receipt of the information. The improved immediate twoway communication process positively impacted alarm management and patient care. Although the voice communication technology did not result in a measurable shortening of time to care delivery for level 1 and 2 alarms, the more complete transfer of alarm information did reduce the requirement for interruption of the care givers' activities and immediate movement to the patient's room in 39\% of alarms, thus improving efficiency of care delivery.

\section{Funding Sources}

This trial was supported by the Dominic and Francine Moceri Fund for Heart Rhythm Research.

\section{Disclosure Statement}

K.A.B., R.I.G., B.M.O., R.D.E., M.A.B., S.D.B., R.S., A.M.S.-none; D.E.H.-Boston Scientific Corp., St. Jude Medical, CardioFocus, Bard Corp (research-modest support) and Contact Surgical, Inc. (equity interest).

\section{REFERENCES}

1. ECG Committee, Subcommittees and Task Forces of the American Heart Association. 2005 American Heart Association guidelines for cardiopulmonary resuscitation and emergency cardiovascular care. Circulation 2005;112: IV12-IV18.

2. Ewy GA, Ornato JP. 31st Bethesda Conference: Emergency cardiac care-task force 1: cardiac arrest. J Am Coll Cardiol 2000;35:832-846.

3. Cummins RO, Ornato JP, Thies WH, Pepe PE. Improving survival from sudden cardiac arrest: The "chain of survival" concept: A statement for health professionals from the Advanced Cardiac Life Support Subcommittee and the
Emergency Cardiac Care Committee, American Heart Association. Circulation 1991;83:1832-1847.

4. Chan PS, Krumholz HM, Nichol G, Nallamothu BK. American Heart Association National Registry of Cardiopulmonary Resuscitation Investigators. Delayed time to defibrillation after in-hospital cardiac arrest. N Engl J Med 2008;358:9-17.

5. Herlitz J, Aune S, Bang A, Fredriksson M, Thoren AB, Ekstrom L. Holmberg S. Very high survival among patients defibrillated at an early stage after in-hospital ventricular fibrillation on wards with and without monitoring facilities. Resuscitation 2005;66:159-166.

6. Eisenberg MS, Mengert TJ. Cardiac resuscitation. N Engl J Med 2001;344: 1304-1313.

7. Ballew KA, Philbrick JT. Causes of variation in reported in-hospital CPR survival: A critical review. Resuscitation 1995;30:203-215.

8. Peberdy MA, Kaye W, Ornato JP, et al. Cardiopulmonary resuscitation of adults in the hospital: A report of 14720 cardiac arrests from the National Registry of Cardiopulmonary Resuscitation. Resuscitation 2003;58:297-308.

9. Lipskis DJ, Dannehl KN, Silverman ME. Value of radiotelemetry in a community hospital. Am J Cardiol 1984;53:1284-1287.

10. Drew BJ, Califf RM, Funk M, et al. Practice standards for electrocardiographic monitoring in hospital settings. Circulation 2004;110:2721-2746.

11. Benezet-Mazuecos J, Ibanez B, Rubio JM, et al. Utility of in-hospital cardiac remote telemetry in patients with unexplained syncope. Europace 2007; 9:1196-1201.

12. Reilly T, Humbrecht D. A nurse-managed remote telemetry model. Crit Care Nurse 2007;27:22-33.

13. Breslin S, Greskovich W, Turisco F. Wireless technology improves nursing workflow and communications. Comput Informat Nursing 2004;22:275-281.

14. Joint Commission on Accreditation of Healthcare Organizations. National Patient Safety Goals 2006. Available at www.jointcommission.org/ (last accessed February 22, 2009).

15. Gee T. An Alarming Matter. 24×7, November 2005:50.

16. Katz MH, Schroeder SA. The sounds of the hospital paging patterns in three teaching hospitals. N Engl J Med 1988;319:1585-1589.

17. Vincent CA, Wears RL. Communication in the emergency department: Separating the signal from the noise. Med J Aust 2002;176:409-410.

18. Glumm MM, Kehring KL, White TL. Effects of visual and auditory cues about threat location on target acquisition and attention to auditory communications. Proc Human Factors Ergonom Soc Mee 2005; 49:347.

19. Gee T. Trends in Point-of-Care Alarm Notification. Patient Safety and Quality Healthcare 2007. Available at http://psqh.com/janfeb07/pointofcare.html (last accessed February 22, 2009).

Address correspondence to: David E. Haines, M.D.

Department of Cardiovascular Medicine Beaumont Hospital

OUWB School of Medicine 3601 W 13 Mile Road Royal Oak, MI 48073

E-mail:dhaines@beaumont.edu

Received: August 17, 2010 Accepted: November 15, 2010 